\title{
LITIGATION SUCCESS FUNCTIONS
}

\author{
Jef De Mot \\ University of Ghent \\ Postdoctoral Researcher FWO
}

\section{Introduction}

Law and economics scholars have traditionally focused more on the reasons for trial than on the trial itself. More recently however, some studies have paid more attention to the legal battle itself. These studies have analyzed the impact of legal rules on the expenditure decisions of the parties at trial and have examined how these effects may change some standard results of the literature. Many of these articles use an explicit function for the probability of a plaintiff victory. These litigation success functions usually take the following form:

$$
p(X, Y)=\frac{X^{a} F}{X^{a} F+Y^{a}(1-F)}=\frac{\left(\frac{X}{Y}\right)^{a} F}{\left(\frac{X}{Y}\right)^{a} F+(1-F)}
$$

with $X$ and $Y$ the expenditures of the plaintiff and the defendant respectively, $F$ the degree of defendant fault (with $0 \leq \mathrm{F} \leq 1$ ), and a the force exponent that indicates the relative importance of expenditures visà-vis the defendant's fault (or briefly, a productivity parameter of the expenditures). This function obviously has the following features (see Hirshleifer and Osborne, 2001): (1) The determinants of success include both the degree of defendant fault and the litigation effort of the parties; (2) the probability of a plaintiff victory depends on the ratio of the effort levels, hence the name "ratio form" that is used for this functional form ${ }^{1}$; (3) For a very close case $(F=1 / 2)$, the outcome at trial depends only upon the litigation efforts; (4) Given equal efforts, the outcome depends only on the degree of fault; (5) If the defendant is totally in the wrong $(F=1)$, she always loses as long as the plaintiff makes some effort $(X>0)$ and (6) If the defendant is totally without fault $(F=0)$, she always wins as long as she makes some effort $(Y>0)$.

Some authors have used a simplified version of this function such as $p(X, Y)=\frac{X F}{X F+Y(1-F)}$, implicitly assuming that $\mathrm{a}=1$ (see e.g. Friehe, 2010), or $p(X, Y)=\frac{X}{X+Y}$, implicitly assuming that $\mathrm{F}=1 / 2$ and $\mathrm{a}=1$ (see e.g. Parisi, 2002).

Some other authors (e.g. Katz, 1988; Farmer and Pecorino, 1999) use a slightly different function:

\footnotetext{
${ }^{1}$ This form was used by Tullock (1980) and the ensuing literature on rent seeking.
} 


$$
p(X, Y)=\frac{e^{M} X^{a}}{e^{M} X^{a}+Y^{a}},
$$

with $M$ reflecting the objective merits of the case (with $M$ varying between $-\infty$ and $+\infty$ ). When $M>0$, the merits favor the plaintiff, when $M<0$ the merits favor the defendant, and when $M=0$ they favor neither party. However, it's easy to see that both functions are equivalent. We can see this by setting $F$ equal to $\frac{e^{M}}{e^{M}+1}$. When $\mathrm{M}=-\infty, \mathrm{F}=0$, when $\mathrm{M}=0, \mathrm{~F}=1 / 2$, and when $\mathrm{M}=+\infty, \mathrm{F}=1$.

This chapter unfolds as follows. Section 2 discusses some results of the literature that uses these litigation success functions. Section 3 examines strengths and weaknesses of these functions and section 4 concludes.

\section{Some results of the literature}

In this section, we first look at the main results of the basic model (2.1). Then we consider an extension: a comparison of adversarial and inquisitorial systems of adjudication (2.2).

\subsection{Basic model: determinants of the expenditures and plaintiff success rate}

In the basic model, both parties select their level of legal expenditures once and simultaneously. The expenditures are investments made to persuade a fact-finder. The amount at stake equals J. Both parties are assumed to be risk-neutral. Each contender aims to maximize his expected income. Each party is assumed to be responsible for his or her own legal expenses regardless of the outcome (the American rule of cost allocation applies). The actual merit of the claim is known by both litigants but is unknown to the court. We obtain solutions for the litigation efforts under the Nash-Cournot protocol.

The plaintiff wants to maximize his expected value:

$$
E V_{p l}=\frac{X^{a} F}{X^{a} F+Y^{a}(1-F)} J-X
$$

The defendant wants to minimize his expected loss:

$$
E V_{d e f}=\frac{X^{a} F}{X^{a} F+Y^{a}(1-F)} J+Y
$$

The first-order conditions are: 


$$
\begin{aligned}
& a F(1-F) X^{a-1} Y^{a} J=\left(X^{a} F+Y^{a}(1-F)\right)^{2} \\
& a F(1-F) X^{a} Y^{a-1} J=\left(X^{a} F+Y^{a}(1-F)\right)^{2}
\end{aligned}
$$

From these conditions, it follows that the parties always spend an equal amount regardless of the level of fault $\left(X^{*}=Y^{*}\right.$ for all $\left.F\right)$. We can now easily find the equilibrium expenditures of the parties:

$$
X^{*}=Y^{*}=a F(1-F) J
$$

The expenditures of the parties increase with the productivity parameter a and with the amount at stake J. They are highest for close cases $(F=1 / 2)$ and lowest for cases with extreme high or low merit $(F=0$ and $F=1)$. In the figure below, the $x$-axis represents $F$, the $y$-axis a and the $z$-axis the expenditures of one of the parties ( $J$ was set equal to $1, F$ varies between 0 and 1 and a between 0 and 3 ).

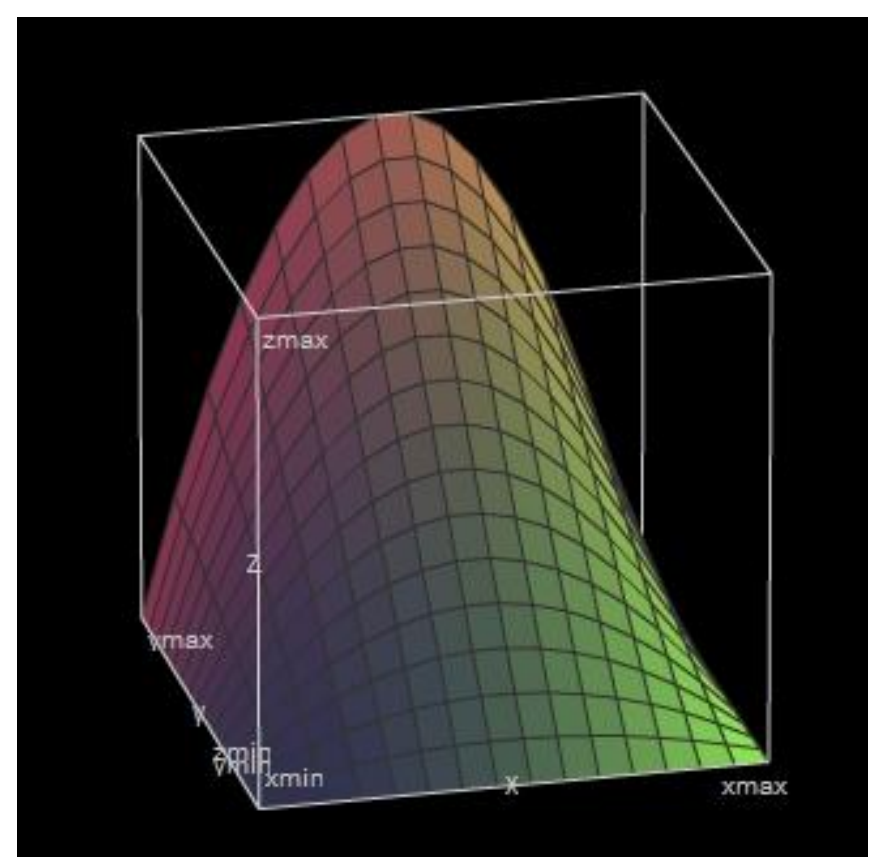

The plaintiff's probability of winning equals $P_{p l}\left(X^{*}, Y^{*}\right)=F$ and thus equals the exogenous quality of the case.

The expected value of the plaintiff and the expected loss of the defendant equal respectively:

$$
\begin{aligned}
& E V_{p l}=F J-a F(1-F) J \\
& E L_{d e f}=F J+a F(1-F) J
\end{aligned}
$$


The plaintiff's expected value is always positive for relatively low values of a, but can be negative for larger values of $a$. The green area in the figure below represents combinations of $F$ and a for which the plaintiff's expected value is positive. The $x$-axis represents $F$ and the $y$-axis a.

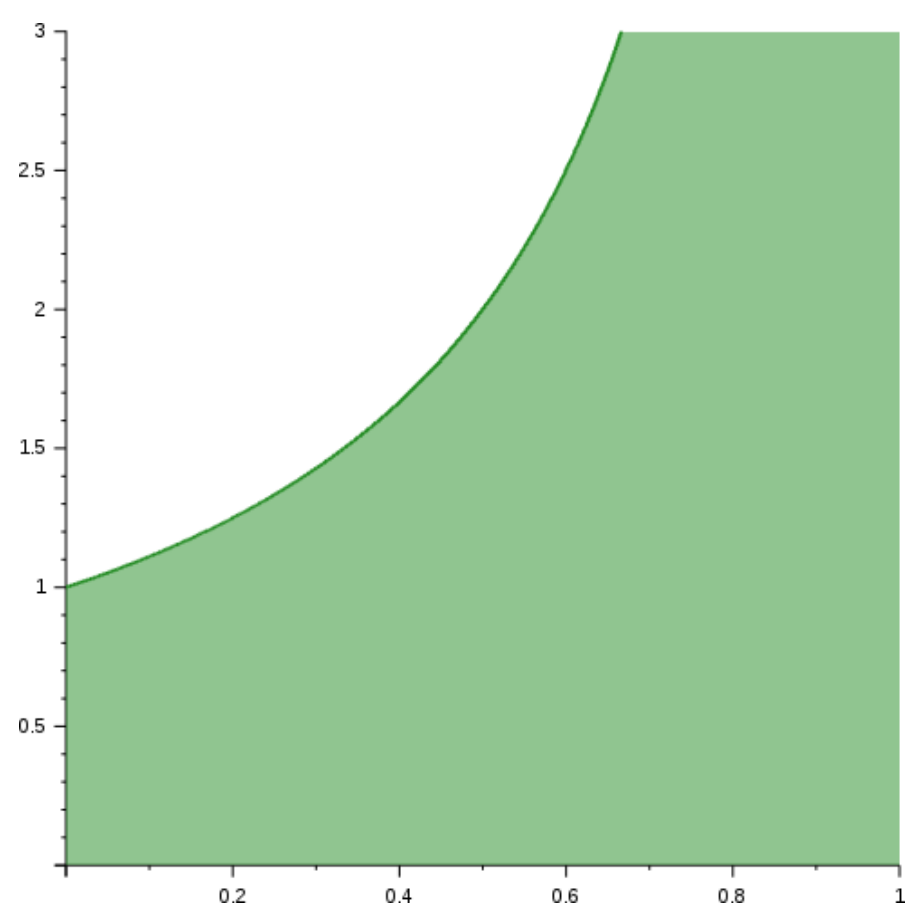

\subsection{Adversarial versus inquisitorial systems}

Parisi (2002) compares the litigation expenditures in an adversarial and an inquisitorial system of adjudication. In an inquisitorial proceeding, the presiding judge dominates the trial. He determines the order in which evidence is taken and evaluates the content of the gathered evidence. In an adversarial system, the process develops through the efforts of the parties before a passive decision maker who takes a decision on the sole basis of the evidence presented by the parties. Of course, the distinction between adversarial and inquisitorial proceedings embraces many more dimensions of the legal process than the ones described here. To be able to consider the range of real world alternatives without artificial or incomplete distinctions, Parisi considers the adversarial nature of the process as a continuous variable (through the variable I, see further). The simple model shows that the expenditures of the parties are larger in an adversarial system.

The following notations are used:

$X:$ the effort of the plaintiff

$Y$ : the effort of the defendant

$Z$ : the judicial effort exerted by the court in independent investigation and examination of independently obtained evidence.

I: variable that captures the weight attached to the inquisitorial findings in determining the 
size of the award. Greater values of I indicate that the judge, as opposed to the litigants, has greater control over the process or that the evidence obtained directly by the judge is, ceteric paribus, given greater weight than the evidence provided by the parties.

$\mathrm{S}$ : a parameter that denotes the level of scrutiny to which evidence provided by the parties is subjected (e.g. S may symbolize procedural safeguards against the admission of certain types of evidence, like hearsay).

$\mathrm{C}$ : the unit cost of litigation effort ( $\mathrm{C}$ is assumed to be constant) $R_{p}^{e}$ and $R_{d}^{e}$ : the total expected return of the plaintiff and the defendant respectively $R_{I}$ : represents the award level that would be given if no adversarial effort is exerted and is a function of the underlying merits of the case and the judicial discovery efforts $Z$.

$R_{A}$ : represents the award amount that results from the parties' evidence. It is a function of $\mathrm{S}$.

The plaintiff's objective is to maximize:

$$
R_{p}^{e}=I R_{I}(Z)+(1-I) R_{A}(S) \frac{X}{X+Y}-C X
$$

The defendant wishes to minimize:

$$
R_{d}^{e}=-I R_{I}(Z)-(1-I) R_{A}(S) \frac{X}{X+Y}-C Y
$$

The first-order conditions for the optimal levels of effort, $\mathrm{X}^{*}$ and $\mathrm{Y}^{*}$, are respectively:

$$
\begin{aligned}
& \frac{\partial R_{p}^{e}}{\partial X}=(1-I) R_{A}(S) \frac{Y}{(X+Y)^{2}}-C=0 \\
& \frac{\partial R_{d}^{e}}{\partial Y}=(1-I) R_{A}(S) \frac{X}{(X+Y)^{2}}-C=0
\end{aligned}
$$

It's easy to see that $X^{*}=Y^{*}$. We can now find the equilibrium levels of effort:

$\mathrm{X}^{*}=\mathrm{Y}^{*}=\frac{(1-I) R_{A}(S)}{4 C}$

The total expenditures in litigation, at cost $\mathrm{C}$, is:

$\mathrm{C}\left(\mathrm{X}^{*}+\mathrm{Y}^{*}\right)=\frac{(1-I) R_{A}(S)}{2}$

The parties will exert litigation effort in proportion to the value of the adversarial component of the case and the weight attached to adversarial evidence in the decision-making process. Note that in a purely adversarial system, the parties will spend half of the value of the case in litigation. 
This result is in line with some insights from other literature on adversarial and inquisitorial systems. For example, Froeb and Kobayashi (2000) have analogized the fact-finding process in an adversarial system to an "extremal" estimator based on the difference between the most favorable pieces of evidence produced by each party. As a consequence, with the involvement of the judge diminishing, the litigants' differences will surface more noticeably, and we should expect greater total expenditures. Adams (1998) has pointed out that in civil law jurisdictions, trial is not a single continuous event. The active participation of the judge blurs the distinction between trial and pretrial. In a two-stage process (pretrial and trial), given the uncertainty over the usefulness and relevance of each piece of information in the later trial stage, the litigants tend to look for much more evidence than is actually utilized in the trial stage. In civil law jurisdictions however, the court confines the scope of the supply of information by the parties to those issues that are rather obscure to him. This provides the parties with an opportunity to get some preliminary feedback from the court as to the likely relevance of costly information, thus avoiding expenditures that may prove unnecessary at a later stage.

\section{Strengths and weaknesses of the formula}

\subsection{Strengths}

One interesting feature of the ratio form is that it can be derived in four different ways: stochastic, axiomatic, optimally-derived, and microfounded (see e.g. Jia, Skaperdas, and Vaidya, 2011). In stochastic derivations, effort is a noisy contributor to some output and the outcome of the contest is determined by a comparison of the outputs of the players. Axiomatic derivations link combinations of axioms to functional forms. In optimal design derivations, a designer with specific objectives about effort or other variables designs the contest, and the functional form is then a result of such a design. Microfounded derivations derive contest functions by characterizing environments in which they naturally emerge as win probabilities of the contestants (e.g. incomplete information, search-based and Bayesian foundations).

Another strength of the ratio form is that several models using it have produced results that have been empirically confirmed. A first example concerns "strategic reciprocality" (see Katz 1988). To describe this phenomenon, it is necessary to introduce some definitions. A party's effort is called provocative when a marginal increase in her effort leads to an increase in her opponent's effort. A party's effort is called deterring when a marginal increase in her effort leads to a decrease in her opponent's effort. The favorite is the party with a greater than 50 percent probability of winning and the underdog is the party with a less than 50 percent probability of winning. Strategic reciprocality means that at the (Nash) equilibrium one party's effort is provocative and one party's effort is deterring. The models using the ratio form lead to such a result: the effort of the favored party is deterring and the effort of the underdog is provocative. In other words: the underdog will spend less when the favored party increases his effort and the favored party will spend more when the underdog increases his effort. This can be explained intuitively. When the favored party increases his effort, the case becomes (even) less close. It becomes less worthwhile for the underdog to spend more. When the underdog increases his effort, the case becomes a closer one. It becomes more worthwhile for the favorite to spend more. Sheperd (1999) 
has made an empirical study of the economics of pretrial discovery. ${ }^{2}$ His findings are in accordance with strategic reciprocality. The author examined 369 U.S. federal cases in which the attorneys of both sides provided information about the number of days they devoted to seeking discovery. ${ }^{4}$ Further information was available through court docket sheets and written questionnaires. Sheperd finds that plaintiffs and defendants behave very differently when conducting discovery. When the plaintiff engages in excessive discovery ${ }^{5}$, the defendant retaliates. The plaintiff on the contrary retreats when the defendant engages in excessive discovery. ${ }^{6} 78$

A second result that has been empirically validated concerns the influence of an increase in the stakes on the expenditures of the parties. In theoretical models that use the ratio form, an increase in the stakes induces both parties to increase their effort since the marginal value of effort rises. Clearly, $a F(1-F) J$ increases with J. Empirical studies have indeed found a strong correlation between the stakes and the efforts of litigants. Kakalik e.a. (1998) find that "higher stakes are associated with significantly higher total lawyer work hours, significantly higher lawyer work hours on discovery, and significantly longer time to disposition". More exactly, "median total lawyer work hours were more than two and a half times larger for cases with monetary stakes over $\$ 500,000$ than for cases with monetary

\footnotetext{
2 Sheperd, G., 'An empirical study of the economics of pretrial discovery', International Review of Law and Economics, 1999, p. 245-263. Sheperd uses data from a survey that researchers at Columbia University conducted in 1962 and 1963 to assess the consequences of the discovery provisions in the Federal Rules of Civil Procedure. The author argues that the data are still relevant despite their age (over 40 years). This is because the discovery rules have not changed substantially after the survey. Although approximately one third of the states introduced a requirement of automatic disclosure, discovery not within the scope of automatic disclosure is still subject to the 'old' rules. Moreover, about two thirds of states still follow the system without automatic disclosure.
}

${ }^{3}$ During pretrial discovery, the parties can force each other to disclose documents and other evidence relevant for the case.

${ }^{4}$ With exclusion of the days the litigant devoted to responding to the requests of the other party.

${ }^{5}$ Excessive according to the defendant.

${ }^{6}$ Excessive according to the plaintiff.

${ }^{7}$ See Sheperd, G., 'An empirical study of the economics of pretrial discovery', International Review of Law and Economics, 1999, p. 260. Sheperd also looks at 'normal' (contrary to excessive) discovery behavior: plaintiffs choose an amount of discovery that reflects the case's underlying fundamentals, like the amount at stake or the number of factual issues. They do not raise their level of discovery when the defendant increases his discovery effort. The defendant however does not look at the fundamentals of the case, but rather mimics the plaintiff: when the plaintiff chooses one unit of discovery, he chooses one unit of discovery himself.

${ }^{8}$ Note that the result of Sheperd implies that discovery may produce efficiency and justice since the plaintiff bases his discovery amount on the fundamentals of the case. At the same time, the fact that the defendant behaves strategically without looking at the fundamentals of the case directly leads to social waste and injustice. See Sheperd, G., 'An empirical study of the economics of pretrial discovery', International Review of Law and Economics, 1999, p. 263. 
stakes of $\$ 500,000$ or less, while mean total lawyer work hours were almost four times larger". ${ }^{9}$ Willging e.a. (1998) find that "the size of the monetary stakes in the case had the strongest relationship to total litigation costs of any of the characteristics we studied." ${ }^{10}$

Third, in its simplest version $\left(P(X, Y)=\frac{X}{X+Y}\right)$, the model predicts that each party spends one fourth of the amount at stake in litigation expenditures. Also in the more complex case of $P(X, Y)=\frac{F X}{F X+(1-F) Y}$, each party spends approximately one fourth of the amount at stake if the case is a close one $(F \approx 1 / 2)$. In an experimental study, Eastman and Viswanath (2003) analyze the litigation expenditure decisions of parties in a setting which allows litigants to believe that they can outsmart (or be outsmarted by) the other litigant in making spending decisions. Litigants were told that their probability of victory was equal to the ratio of their effective litigation spending to that of the other side. The instructions explained that effective litigation spending could be equal to actual dollars spent, but could also be higher or lower than actual spending. The litigants were told that the effectiveness of their spending would be determined based on an outside evaluation. The case involves a car accident with stipulated damages of $\$ 100.000$. Litigants were given the option of spending on several categories (e.g. legal research, hiring a detective etc.). They were not given the opportunity to settle the case. The authors find that the respondents spent an average of $\$ 30.000$, which is relatively close to and statistically not different from the prediction of rational choice theory $(\$ 25.000)$.

Fourth, the model predicts that the parties will spend the same amount (no matter how low or high the quality of the case). This finds some confirmation in the same experimental study of Eastman and Viswanath (2003). In most cases, the parties spent a similar amount.

\subsection{Weaknesses}

We have seen that the ratio form can be derived in several ways (see 4.1). However, other functional forms can also be derived in different ways: the logit function and the probit function (see Jia, Skaperdas, and Vaidya, 2011). These three functions differ in their assumptions on the distributions of the error terms. In the Tullock model the error terms have an inverse exponential distribution (Jia, 2008), in the logit model they are extreme value distributed (McFadden, 1974), and in the probit model they are normally distributed. Empirical tests have not yet been successful in showing which model best captures the characteristics of a particular contest and gives the most accurate predictions (see Jia, Skaperdas, and Vaidya, 2011).

\footnotetext{
${ }^{9}$ Kakalik, James S., Hensler, Deborah R., McCaffrey, Daniel, Oshiro, Marian, Pace, Nicholas M. and Vaiana, Mary E., 'Discovery Management: Further Analysis of the Civil Justice Reform Act Evaluation Data', 39 B.C.L. Rev, 1998, p. 613 and 648 .

${ }^{10}$ Willging, Thomas E., Stienstra, Donna, Shapard, John and Miletich, Dean, 'An Empirical Study of Discovery and Disclosure Practice Under the 1993 Federal Rule Amendments', 39 B.C.L. Rev, 1998, p. 532.
} 
Some predictions of the ratio form have not been confirmed. For example, the model predicts that the expenditures of litigants increase linearly with the amount at stake. However, empirical research by Kakalik e.a. (1984) found that the expenditures of the parties do not rise in proportion to the amount awarded, but rather concavely. ${ }^{11}$ This cannot be seen as a fundamental flaw of the ratio form. If we would introduce fixed costs on top of variable costs in the basic model, then the expenditures would not rise linearly with the amount at stake anymore.

Some other criticisms do not explicitly concern the type of function that is used for the plaintiff's probability of victory, but rather the solution concept that is employed. Most articles rely on the NashCournot protocol: the parties choose their expenditures in ignorance of the opponent's simultaneous choice. However, social interactions are usually too complex to be captured by any simple model. In some contexts, other solution concepts may be more satisfactory. For example, under the Stackelberg solution concept, one side commits to a level of effort, to which the other side then makes an optimizing response. Using this solution concept, Hirshleifer and Osborne (2001) find some interesting differences with the model that relies on the Nash-Cournot protocol: the litigation effort of the parties are almost always unequal and the side with the better case tends to fight harder. Outcomes tilt disproportionally in favor of the side with the more meritorious case.

\section{Conclusion}

Specific litigation success functions can be helpful in analyzing the consequences of legal rules. The function most intensively used in the literature, the ratio form, has strong theoretical underpinnings and leads to results which have been empirically validated. However, more theoretical and empirical research is necessary to determine whether other functional forms capture the expenditure decisions for some types of disputes even better.

\footnotetext{
${ }^{11}$ Kakalik, J.S. e.a., Variation in Asbestos Litigation Compensation and Expenses, Rand Corporation, 1984, 91p.
} 